\section{Lossy Network Correlated Data Gathering With High-Resolution Coding}

Răzvan Cristescu, Member, IEEE, and Baltasar Beferull-Lozano, Member, IEEE

\begin{abstract}
Sensor networks measuring correlated data are considered, where the task is to gather data from the network nodes to a sink. A specific scenario is addressed, where data at nodes are lossy coded with high-resolution, and the information measured by the nodes has to be reconstructed at the sink within both certain total and individual distortion bounds. The first problem considered is to find the optimal transmission structure and the rate-distortion allocations at the various spatially located nodes, such as to minimize the total power consumption cost of the network, by assuming fixed nodes positions. The optimal transmission structure is the shortest path tree and the problems of rate and distortion allocation separate in the high-resolution case, namely, first the distortion allocation is found as a function of the transmission structure, and second, for a given distortion allocation, the rate allocation is computed. The second problem addressed is the case when the node positions can be chosen, by finding the optimal node placement for two different targets of interest, namely total power minimization and network lifetime maximization. Finally, a node placement solution that provides a tradeoff between the two metrics is proposed.
\end{abstract}

Index Terms-Energy efficiency, high-resolution rate-distortion, sensor networks, shortest path tree.

\section{INTRODUCTION}

\section{A. Lossy Correlated Data Gathering}

Consider a network of sensors measuring a spatially correlated data field. An important task in such a scenario is data gathering, where the goal is to gather data from all the nodes to the base station, or the sink, via a subset of the links of the network. An example is shown in Fig. 1, where there are $N$ nodes with sources $X_{1}, \ldots, X_{N}$, a sink denoted by $S$, and a graph of connectivity among the nodes. The transmission topology is assumed to be an undirected connected graph, with nodes within a certain transmission range connected by point-to-point links. Data at nodes have to be encoded for proper reconstruction of the field at the sink node within a certain prescribed total distortion (in Fig. 1, we denote the distortion and rate at node $i$ by $D_{i}$ and $R_{i}$, respectively). In scenarios where a minimum accuracy is necessary for individual measurements across network nodes, there are also additional distortion upper bounds imposed on the individual data.

In addition to encoding of the data by the nodes, these data also need to be transmitted over the network from the sources to the sink, which results in certain transmission costs (in Fig. 1, the total weight of the path from node $i$ to the sink is denoted by $c_{i}$ ). We study the interplay between the rate-distortion allocations at nodes, and the transmission structure used to transport the data, by means of cost functions that are functions of both. The cost functions usually found in practice separate the rate term from the path weight term [5]. For instance, the

Manuscript received March 12, 2005; revised February 8, 2006. The material in this correspondence was presented in part at IPSN 2005, Los Angeles, CA, April 2005.

R. Cristescu was with the Center for Mathematics of Information, California Institute of Technology, Pasadena, CA USA. He is now with Beckton Dickinson and Co., Sparks, MD 21152 USA.

B. Beferull-Lozano was with the Audiovisual Communications Laboratory, Swiss Federal Institute of Technology (EPFL), Lausanne, Switzerland. He is now with Universidad de Valencia, Instituto de Robótica-School of Engineering, Group of Information and Communication Systems, 46980, Paterna (Valencia), Spain.

Communicated by R. W. Yeung, Guest Editor.

Digital Object Identifier 10.1109/TIT.2006.874536

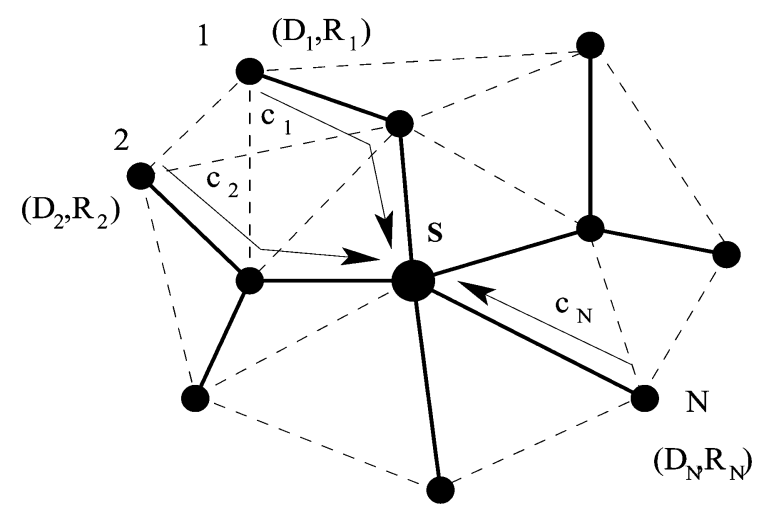

Fig. 1. Data from nodes $1,2, \ldots, N$ need to arrive at sink $S$. A rate $R_{i}$ with corresponding distortion $D_{i}$ is allocated to each node $i$. In thick solid lines, a chosen tree transmission structure is shown; in thin dashed lines, the other possible links are shown. The weight of the path from node $i$ to the $\operatorname{sink}$ is $c_{i}$, and the corresponding path is shown in gray line.

(rate) $\times$ (path weight) cost function measures the power consumption in sensor networks, where (rate) is the number of transmitted bits, and (path weight) is a supraunitary power $\kappa$ (usually $\kappa=2$ or $\kappa=4$ ) of the Euclidean distance over which transmission is done. Typical goals in such scenarios are: a) the minimization of the total network power consumption, defined as the total sum of powers consumed by nodes, and b) maximization of the network lifetime, defined as the time until the first node fails (it runs out of power). We consider jointly the optimization of both rate and distortion allocation at nodes, transmission structure, and node placement, in the context of sensor networks measuring correlated data.

Let $\mathbf{X}=\left(X_{1}, \ldots, X_{N}\right)$ be the vector formed by the random variables representing the sources measured at nodes $1, \ldots, N$. The samples taken at nodes are temporally independent and identically distributed (i.i.d.) $)^{1}$ and spatially correlated. We assume that the random variables are continuous and that there is a high-resolution multidimensional quantizer in each sensor. A rate-distortion allocation $\left\{\left(R_{1}, D_{1}\right), \ldots,\left(R_{N}, D_{N}\right)\right\}$ (bits) has to be assigned at the nodes so that the quantized measured information samples are described within a certain total distortion $D$ and individual distortion upper bounds $D_{i}^{\max }, i=1, \ldots, N$. That information is transmitted through the links of the network to the designated sink node ${ }^{2}$ (see Fig. 1). In [8], [10], the problem of lossless coding of the data at nodes with Slepian-Wolf coding [19] has been studied. In this work, we extend that problem to the case when nodes code their data in a lossy manner, such that the information received by the sink describes the data at nodes within certain prescribed distortion constraints. Namely, the rate allocation essentially depends on the differential entropy of the nonquantized data that is measured and on the distortion levels of quantization that are assigned to each of the sources located at nodes.

Moreover, we study the problem of optimal node placement for the tasks of first, minimizing the total network power consumption, and second, maximizing the network lifetime. Note that the placement solutions resulting from these two corresponding optimizations are different, since optimizing the lifetime of the network means equalizing power consumption at nodes, which does not necessarily coincide with the optimal placement solution when the sum of power consumptions

\footnotetext{
${ }^{1}$ In the case of temporally correlated Gaussian sources, i.i.d. sources can be obtained by applying locally at each sensor a linear decorrelation transform.

${ }^{2}$ Due to the interaction between the rate-distortion region and the transmission structure optimization, our results can be easily generalized to the case of multiple sinks, as we will show in this correspondence.
} 
has to be minimized. Interestingly, for the correlation functions we consider in this correspondence, when the total power consumption is minimized the bottleneck node in the optimal positioning is the one at the extremity of the network, rather than the node closest to the sink. This is both due to the coding strategy, that assigns low rates to nodes far from the sink, and to the internode distance optimization, as we show in this correspondence.

The general distributed rate-distortion region for coding correlated data at general quantization resolution levels (e.g., low rates) is unknown even for the Gaussian case [3], [16]. Thus, we will restrict our study to the case of data gathering with lossy high-resolution quantization for arbitrarily correlated sources [21], where the rate-distortion region is known. This assumption allows for the separation between rate allocation and transmission structure optimization, which is a simplification over the deep and difficult problem of low resolution coding, for which reencoding at nodes can help in reducing the coding rates [20]. In general, the region obtained for the high-resolution case provides an outer bound, which becomes tighter as the resolution increases. Thus, the results we obtain for the data gathering scenario in the high-resolution case provide a lower bound on the performance of any other rate-distortion allocation strategy, in terms of power efficiency, for a network that performs data gathering of correlated data. Moreover, our results on the problem of joint rate-distortion allocation and transmission structure optimization can be generalized should good bounds for low-resolution rate-distortion curves become available.

\section{B. Related Work}

Recent work that exploit correlation in the data in the context of sensor networks include [2], [11], [15], [17]. A setting related to the problem we consider in this correspondence, namely optimizing correlated data gathering with communication costs for the case of lossless coding of finely quantized data with the same scalar quantizer at each node, has been studied in [8], where Slepian-Wolf [6], [19] bounds are used, and in [9], where a simple coding technique with explicit communication is considered; in this work, while assuming high-rate quantization, lattice quantizers are used where the resolutions of the quantizers can be adapted across nodes.

A similar problem of data compression by opportunistic aggregation was considered in [14], where no collaboration among nodes is assumed. In this work we consider collaboration among nodes by lossy coding of jointly correlated data at nodes, and we provide the optimal allocation within the rate-distortion region given in [21], that minimizes the transport cost to perform the data gathering to a sink. Moreover, we consider the effect of this coding technique on the optimal placement of the nodes for gathering lossy coded data when both total network power efficiency and lifetime optimization are considered.

\section{Main Contributions and Outline}

We solve the total power efficiency optimization problem by finding the transmission structure and the rate-distortion allocations across nodes in a closed form, for high-resolution coding of correlated data. Namely, we show that: a) the shortest path tree $(S P T)$ is the optimal transmission structure, and b) the solution of the rate-allocation optimization can be decoupled in two steps: first find the rate allocation, and then use the closed-form solution for the rate allocation to determine the distortion allocation. Our result also provides a general lower bound in terms of power efficiency for the cost of data gathering of lossy coded data in sensor networks with a given connectivity graph. Further, when the positions of the nodes can be chosen, we determine the optimal node placement for optimizing two metrics of interest, namely the total power consumption and the network lifetime. Finally, we propose a solution for the node placement which provides a tradeoff between the two metrics.
The rest of this correspondence is structured as follows. In Section II, we formulate the optimization problem considered in this correspondence, and briefly introduce a family of Gaussian random fields that we use as an example of correlation fields. In Section III, we show that the optimization problem separates, and we sequentially find the optimal transmission structure, rate allocation, and distortion allocation under various settings with total and individual distortion constraints. Further, in Section IV, we find the optimal placement in a one-dimensional (1-D) scenario for two optimization goals, namely network total power and network lifetime, and we present numerical simulations to illustrate our results. We also propose a simple solution for the node placement that provides a tradeoff between the two metrics. We conclude with Section V.

\section{PRoblem Setting}

\section{A. Optimization Problem}

For the optimization of rate-distortion allocation and transmission structure, we use a family of cost functions that separate the rate and the total path weights. These power cost functions are relevant for various problems in sensor networks related to power efficiency optimization [5]. More specifically, we assume that the power to transmit a bit through a link distance $w_{e}$ is $w_{e}^{\kappa}, \kappa \geq 2$. Our results also apply for other models for the power dependence on the distance [12].

Note that, as opposed to the Slepian-Wolf scenario considered in [8], lossy coding of data implies an additional number of optimization variables, namely the values of the distortions $\left\{D_{i}\right\}_{i=1}^{N}$ at nodes. This increases significantly the complexity of the problem. Additional constraints are introduced by the rate-distortion region [21], which is similar in form to the Slepian-Wolf region [19], but includes additional terms related to the various distortions. Moreover, as in the Slepian-Wolf scenario, no communication is required among the nodes for data coding. As a result of this, the problems of transmission structure optimization, rate, and distortion allocation separate, as we will see in detail in Section III.

Assume a given node placement such as the one illustrated in Fig. 1. Denote $R_{i}$ and $D_{i}, i=1 \ldots N$, the allocated rate and distortion at node $i$. The most general form of our optimization problem is given as follows:

$$
\left\{R_{i}^{*}, D_{i}^{*}, S T^{*}\right\}_{i=1}^{N}=\arg \min _{\left\{R_{i}, D_{i}, S T\right\}_{i=1}^{N}} \sum_{i=1}^{N} c_{i} R_{i}
$$

under the constraints

$$
\begin{aligned}
\sum_{i \in \mathcal{X}} R_{i} & \geq h(\mathcal{X} \mid V \backslash \mathcal{X})-\log (2 \pi e)^{|\mathcal{X}|} \prod_{i \in \mathcal{X}} D_{i}, \forall \mathcal{X} \subseteq V \\
\sum_{i=1}^{N} D_{i} & \leq D \\
D_{i} & \leq D_{i}^{\max }, \quad i=1 \ldots N
\end{aligned}
$$

where $S T$ is a spanning tree, which defines the transmission structure; $c_{i}, i=1 \ldots N$ are the total weights of the path from node $i$ to the sink on the spanning tree $S T$, thus $c_{i}=\sum_{e \in \mathcal{E}_{i}} w_{e}^{\kappa}$, where $e \in \mathcal{E}_{i}$, and $\mathcal{E}_{i}$ is the set of edges linking node $i$ to the sink $S$ on $S T$, and $w_{e}$ is the Euclidean distance of edge $e ; h(\cdot)$ denotes the differential entropy. Constraints (2) express the rate-distortion region in [21], namely the sum of rates for any given subset of nodes is larger than the entropy of the random variables measured at those nodes, conditioned on the random variables measured at all other nodes. In constraint (3), $D$ is the maximum total distortion and in (4), $D_{i}^{\max }, i=1 \ldots N$ are the maximum individual constraints. 
The optimization problem (1) assumes a fixed placement of nodes. However, in practice, a well chosen placement of nodes (namely, allowing adjustable values of $c_{i}$, under the constraint that the desired measured field is fully covered by the nodes) can drastically increase the total efficiency of the network, as shown in [13]. In the general 2-D setting, (1) is hard to formalize and solve when the node placement is introduced as a variable in the optimization, since a proper constraint set for the node placements, that preserves coverage, needs to be defined. Thus, in Section III, where we study the general optimization for a 2-D network, we will consider that the node placement is fixed. However, in Section IV, we will consider the optimization (1) in the 1-D case, for which we additionally solve the placement problem. Namely, we provide optimal node placements for: a) minimization of the total power consumed by the network, and b) maximization of the lifetime of the network.

\section{B. Example: Correlated Gaussian Random Field}

For the sake of clarity, in our numerical simulations we assume a jointly Gaussian model for the spatial data $\mathbf{X}$ measured at nodes, with an $N$-dimensional multivariate normal distribution $\mathbf{X} \sim \mathcal{N}^{N}(\mu, \mathbf{K})$

$$
f(\mathbf{X})=\frac{1}{\sqrt{2 \pi} \operatorname{det}(\mathbf{K})^{1 / 2}} e^{-\left(\frac{1}{2}(\mathbf{X}-\mu)^{T} \mathbf{K}^{-1}(\mathbf{X}-\mu)\right)}
$$

where $\mathbf{K}$ is the covariance matrix of $\mathbf{X}$, and $\mu$ the mean vector. The diagonal elements of $\mathbf{K}$ are the variances $K_{i i}=\sigma_{i}^{2}$. The rest of $K_{i j}$ depend on the distance between the corresponding nodes (e.g., $K_{i j}=$ $\sigma_{i i} \sigma_{j j} \exp \left(-a d_{i, j}^{\beta}\right)$ or $K_{i j}=\frac{\sigma_{i i} \sigma_{j j}}{1+a d^{\beta}}$, with $a>0$ and $\left.\beta \in\{1,2\}\right)$, where $d_{i, j}$ is the distance between nodes $i$ and $j$ [7], [10]. Without loss of generality, we will restrict our analysis to unit variance $\sigma_{i i}=1$, $i=1 \ldots N$ and zero-mean $\mu=0$ processes: $\mathbf{X} \sim \mathcal{N}^{N}(0, \mathbf{K})$.

Note that although the numerical evaluations will be performed using the Gaussian random field model, our results are valid for any spatially correlated random processes, whose correlation decreases with the distance.

\section{OPTIMIZATION}

\section{A. Optimal Transmission Structure and Rate Allocation}

Constraints (2) imply that nodes can code (asymptotically at high resolution) with any rate that obeys the rate-distortion region without explicitly communicating data with each other. As a consequence, the optimization problem (1) can be achieved by first optimizing the transmission structure with respect to only the path weights $c_{i}$, which implies that the optimal transmission structure is the shortest path tree $S T^{*}=S P T$ [8]. Denote by $c_{i}^{*}$ the total weight of the path from node $i$ to the sink $S$ on the $S P T$. Suppose without loss of generality that nodes are ordered in a list with increasing values of the weights $c_{1}^{*} \leq c_{2}^{*} \leq \cdots \leq c_{N}^{*}$. Then the optimization (1) becomes

$$
\left\{R_{i}^{*}, D_{i}^{*}\right\}_{i=1}^{N}=\arg \min _{\left\{R_{i}, D_{i}\right\}_{i=1}^{N}} \sum_{i=1}^{N} c_{i}^{*} R_{i}
$$$$
\text { under the constraints (2)-(4). }
$$

Our results can be extended to the case of multiple sinks, by replacing the $S P T$ with a superpositions of Steiner trees, corresponding to the optimal transmission structure from each node to the corresponding sinks. Therefore, we will restrict our analysis to the case of a single sink.
Next, we show that, regardless of constraints (3), (4), the optimal rate allocation in (5) has to obey only constraints (2); namely, for any set of distortion values $\left\{D_{i}\right\}_{i=1}^{N}$, the rate allocation is given by the following.

Theorem 1: Optimal rate allocation

$$
\begin{aligned}
R_{1}^{*} & =h\left(X_{1}\right)-\log 2 \pi e D_{1} \\
R_{2}^{*} & =h\left(X_{2} \mid X_{1}\right)-\log 2 \pi e D_{2} \\
& \ldots \\
R_{N}^{*} & =h\left(X_{N} \mid X_{N-1}, \ldots, X_{1}\right)-\log 2 \pi e D_{N} .
\end{aligned}
$$

The proof of Theorem 1 uses similar techniques as in [8], and for the sake of conciseness we omit it.

\section{B. Total Distortion Constraint}

Consider optimization of (1) for the case where constraints (4) are inactive. From Theorem 1, if the positions of the nodes are fixed, then $\left\{R_{i}^{*}\right\}_{i=1}^{N}$ only depend on $\left\{D_{i}\right\}_{i=1}^{N}$. Therefore, at this point, we can insert in (5) the values for $\left\{R_{i}^{*}\right\}_{i=1}^{N}$ as given by Theorem 1, thus, obtaining an optimization problem having as argument only the set of distortions $\left\{D_{i}\right\}_{i=1}^{N}$

$\left\{D_{i}^{*}\right\}_{i=1}^{N}=\arg \min _{\left\{D_{i}\right\}_{i=1}^{N}} \sum_{i=1}^{N} c_{i}^{*}\left(h\left(X_{i} \mid X_{i-1}, \ldots, X_{1}\right)-\log 2 \pi e D_{i}\right)$

This can be equivalently written as

$\left\{D_{i}^{*}\right\}_{i=1}^{N}=\arg \max _{\left\{D_{i}\right\}_{i=1}^{N}} \sum_{i=1}^{N} c_{i}^{*} \log 2 \pi e D_{i}$ under the constraint (3).

Denote $\sum_{i=1}^{N} c_{i}^{*}=C$. The solution of the optimization problem (8) is obtained using Lagrange multipliers

$$
D_{i}^{*}=D \cdot \frac{c_{i}^{*}}{C}, \quad i=1 \ldots N .
$$

By combining (6) and (9), the rate-distortion allocation at nodes is given by

$$
R_{i}^{*}=h\left(X_{i} \mid X_{i-1}, \ldots, X_{1}\right)-\log \left(\frac{2 \pi e D c_{i}^{*}}{C}\right), \quad i=1 \ldots N .
$$

For correlation functions that depend only on the distance among nodes and for uniform placement of nodes the differential entropy monotonically decreases as the number of nodes on which conditioning is done increases. Also, by definition, the sequence $\left\{c_{i}^{*}\right\}_{i=1}^{N}$ is monotonically increasing with $i$. As a result, the rate allocation $R_{i}$ in (10) is a function that monotonically decreases with the node index $i$.

Further, (10) depends essentially only on the ordering $\left\{c_{i}^{*}\right\}_{i=1}^{N}$. For example, in the case of correlated Gaussian random fields, (10) can be written as

$$
R_{i}^{*}=\log \frac{\operatorname{det}(\mathbf{K}(1, \ldots i))}{\operatorname{det}(\mathbf{K}[1, \ldots, i-1])} \frac{C}{c_{i}^{*} \cdot D}, \quad i=1 \ldots N
$$

where $\mathbf{K}(1, \ldots, i)$ is the correlation matrix corresponding to nodes $1, \ldots, i$. 


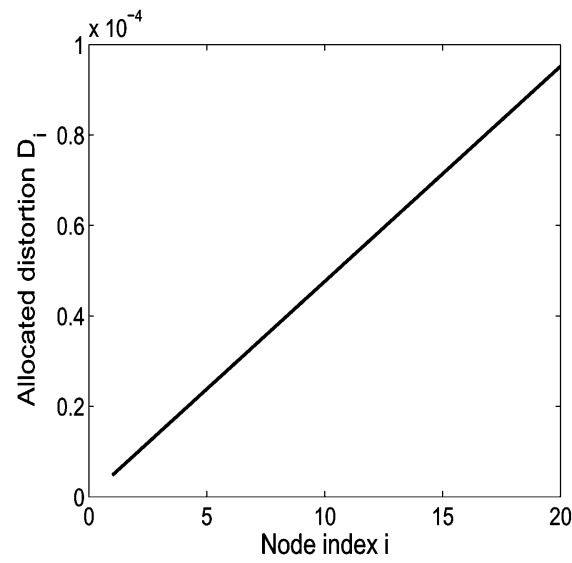

(a)

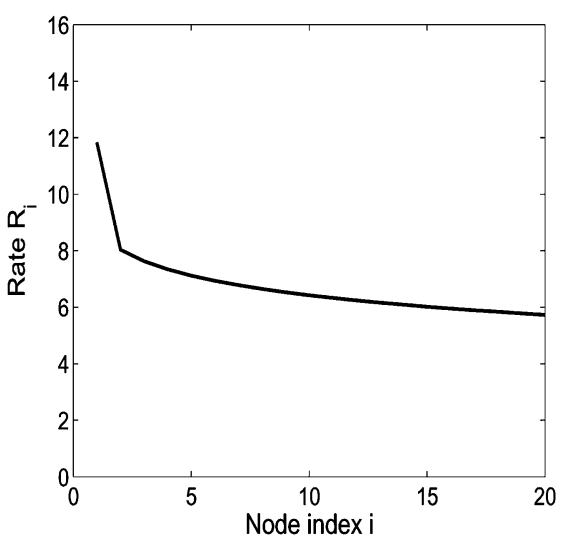

(b)

Fig. 2. A 1-D grid with constraint on the total distortion. Nodes are labeled with increasing indexes, with node 1 closest to the sink and node $N$ farthest. The correlation model is $\exp \left(-a d_{i, j}\right)$. (a) Distortion allocation as a function of node index. (b) Rate allocation as a function of node index.
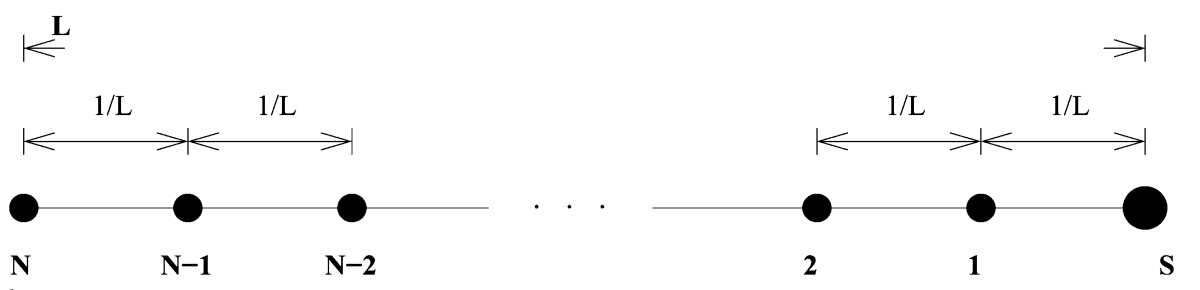

Fig. 3. A 1-D grid network.

In Fig. 2, we illustrate the distortion and rate allocations provided by (11) for the 1-D grid shown in Fig. 3, with uniform internode distances, and measuring a correlated Gaussian random field with $\beta=1$ (which corresponds to sampling a continuous Gauss-Markov process). The same analysis holds for arbitrary 2-D networks. For clarity in our numerical experiments, we keep the values of the model parameters fixed along this correspondence: Gaussian random processes with $\alpha=$ $10^{-3}, \beta=1$, and $N=20, D=10^{-3}, D_{i}^{\max }=0.7 \cdot 10^{-3}, \kappa=2$.

These results are intuitively expected: at the extremities of the network (where the $\operatorname{cost} c_{i}^{*}$ of the path from node $i$ to the sink is large), small rates are allocated, due to both large distortions and conditioning on many nodes; on the contrary, large rates, meaning small distortions and conditioning on fewer nodes, are allocated at nodes near the sink (where the cost $c_{i}^{*}$ is small). In general, the rates increase monotonically as nodes are closer to the sink.

Note that for $\beta=1$, the resulting continuous process is Gauss-Markov, which means that if the nodes are equally-spaced then the differential entropies are equal in (10), and thus the decrease in rate in Fig. 2(b) is due only to the increase in allocated distortion.

Similar results are obtained for many other network parameter settings. For instance, for a process with $\beta=2$, the result is a less abrupt decay of the rate when the node index increases, as a result of the smoother decrease in differential entropy and increase in allocated distortion in (11), with the number of nodes on which conditioning is done (see Fig. 4, for $N=20, D=10^{-3}, \alpha=0.1, \kappa=2, \beta=2$ ). For the sake of clarity, in what follows, we only show numerical results for processes with correlation function $\exp (-a|d|)$; the curves corresponding to processes with correlation function $\exp \left(-a|d|^{2}\right)$ or $\frac{1}{1+a|d|^{\beta}}$ are similar in shape and behavior.

\section{Individual Distortion Constraints}

Consider now additionally the individual constraints (4). For the sake of clarity, assume first that all individual constraints are equal, namely $D_{i}^{\max }=T$. Using Lagrange optimization, the optimal solution can be obtained as follows.

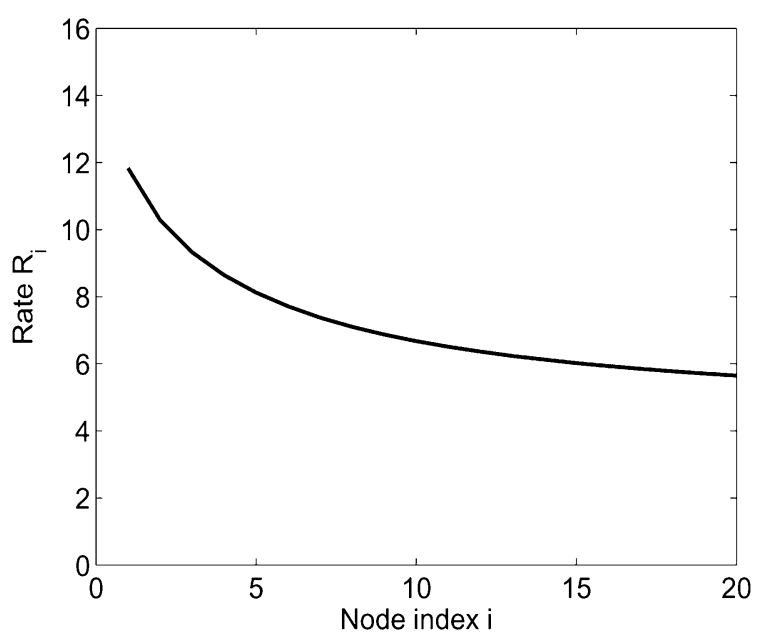

Fig. 4. Optimal rate allocation for the 1-D grid with constraint on the total distortion. The correlation model is $\exp \left(-a d_{i, j}^{2}\right)$.

- Consider the solution for the distortion allocation given by (9), without introducing individual distortion constraints.

- Let $m+1$ be the smallest node index for which $D_{i} \geq T, i=$ $m+1 \ldots N$. Allocate $D_{i}^{*}=T, i=m+1, \ldots, N$, that is, the last $N-m$ constraints are active. For the rest of the distortion values $D_{i}, i=1, \ldots, m$, compute

$$
\left\{D_{i}^{*}\right\}_{i=1}^{m}=\arg \max _{\left\{D_{i}\right\}_{i=1}^{m}} \sum_{i=1}^{m} c_{i}^{*} \log 2 \pi e D_{i}
$$

under the constraint

$$
\sum_{i=1}^{m} D_{i} \leq D-(N-m) T
$$




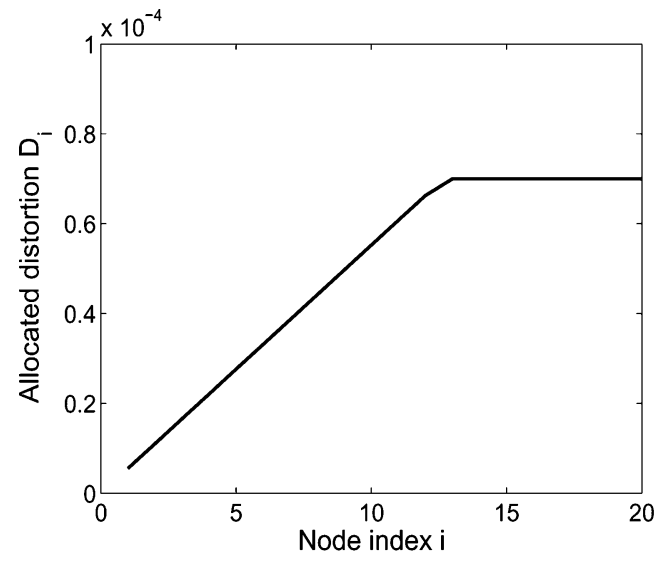

(a)

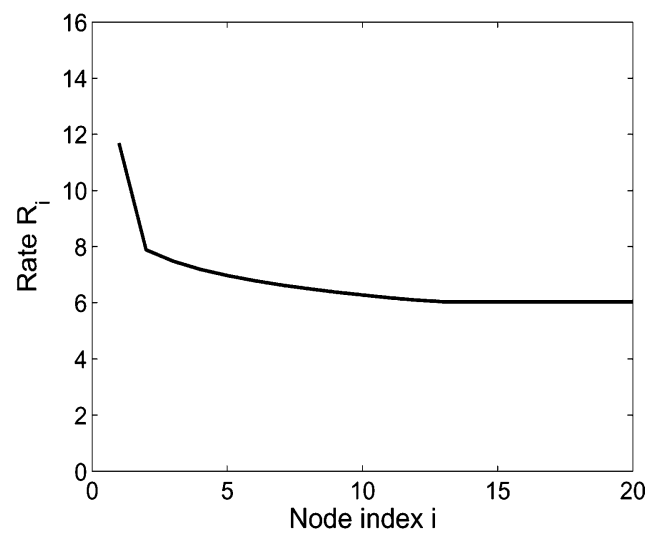

(b)

Fig. 5. 1-D grid with constraints on the total and individual distortions. Nodes are labeled with increasing indexes, with node 1 closest to the sink and node $N$ farthest. (a) Distortion allocation as function of node index. (b) Rate allocation as a function of node index.

which is solved similarly to (8). Thus, the complete solution is readily given by

$$
\begin{aligned}
& D_{i}=T, \quad i=m+1 \ldots N \\
& D_{i}=c_{i}^{*} \frac{D-(N-m) T}{C_{m}}
\end{aligned}
$$

where $C_{m}=\sum_{i=1}^{m} c_{i}^{*}$.

Fig. 5 shows the distortion and rate allocations obtained in this case. Fig. 5(b) illustrates that when individual distortions are considered, the rates allocated at the nodes of the extremity of the network are equalized [as compared to Fig. 2(b)]. As a result of the rate load added to nodes at the extremity of the network, the rates at the nodes closer to the sink are correspondingly decreased.

The generalization to the case of nonequal individual constraints is straightforward.

\section{Placement Optimization FOR THE 1-D NETWORK}

\section{A. Total Power Minimization}

In some scenarios, the positions of the nodes are not fixed in advance, and nodes may be placed optimally so that to minimize various resources [13] (e.g., total power efficiency). Here, we consider the optimization (1) where the positions of the nodes are variables, and the optimization is done additionally over the weights of the paths from the nodes to the sink

$$
\left\{R_{i}^{*}, D_{i}^{*}, c_{i}^{*}\right\}_{i=1}^{N}=\arg \min _{\left\{R_{i}, D_{i}, c_{i}\right\}_{i=1}^{N}} \sum_{\substack{i=1 \\ \text { under the constraints (2)-(4) }}}^{N} c_{i} R_{i}
$$

with additional constraints on $\max \left\{c_{i}\right\}_{i=1}^{N}$ (coverage constraint) and on $\max \left\{c_{i}-c_{i-1}\right\}_{i=2}^{N}$ (internode space constraint). The coverage constraint imposes that the entire area is covered. The internode space constraint ensures that any given point in the measured area is close enough to a sensor node, such that the data corresponding to that point can be reconstructed with a certain minimal accuracy at the sink, by approximating it with the value measured by the closest sensor.

For the sake of simplicity, here, we consider a 1-D scenario (see Fig. 3). A similar analysis can be performed in the 2-D case, since the rate and distortion allocations depend only on the ordering nodes on the

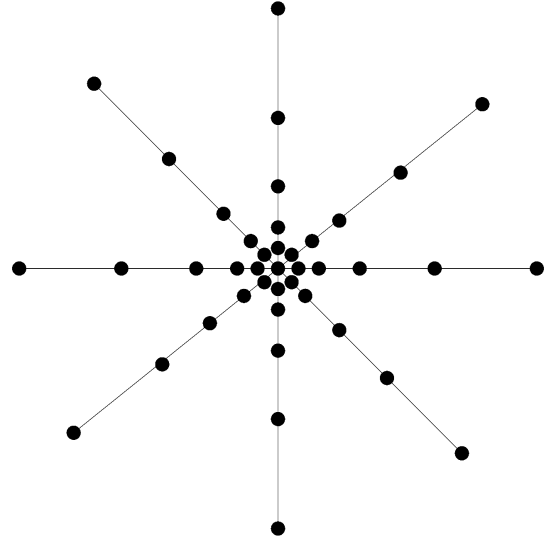

Fig. 6. A wheel 2-D placement, with the sink in the center.

$S P T$; however, in the 2-D case, an additional model describing how nodes can be moved from their initial positions is required (e.g., on 2 -D grids), which makes the problem more complicated. One possible solution is to use a radial structure for the node placement, as in [13] (see Fig. 6), where the placement of nodes for each spoke is as in the optimal 1-D placement presented in this section. A complete study of the optimal number of spokes and number of nodes per spoke for a given $N$ is beyond the scope of this correspondence.

Finding analytically the solution for (13) by partial derivatives is complicated, since the expressions for the rates $\left\{R_{i}^{*}\right\}_{i=1}^{N}$ obtained in (10) depend on both $\left\{D_{i}^{*}\right\}_{i=1}^{N}$ and $\left\{h\left(X_{i} \mid X_{i-1}, \ldots, X_{1}\right)\right\}_{i=1}^{N}$; moreover, these sets of values depend themselves on the values of $\left\{c_{i}\right\}_{i=1}^{N}$. Instead, we use the following iterative algorithm for optimization.

Algorithm 1: Optimal Placement (see Fig. 7)

- Step 1) Initially, the values of $c_{i}$ are chosen such that the nodes are equally spaced (as in Fig. 3 ). ${ }^{3}$

- Step 2) until convergence do:

- a) Solve (13) with parameters $\left\{c_{i}\right\}_{i=1}^{N}$ to obtain $\left\{R_{i}\right\}_{i=1}^{N}$ as in (10).

- b) Solve (13) with parameters $\left\{R_{i}\right\}_{i=1}^{N}$ to obtain $\left\{c_{i}\right\}_{i=1}^{N}$.

The convergence of the algorithm is ensured by the convexity of the cost function and the constraint sets [4]. Namely, the cost function is

${ }^{3}$ Note that the placement uniquely determines the ordering of nodes. Namely, once an order is set among the nodes, the same order will hold along the optimization, while only the distances between the nodes will change. 


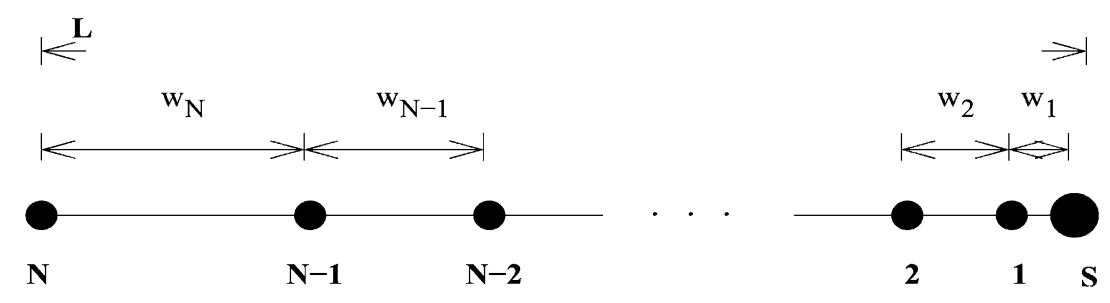

Fig. 7. Optimized placement for the 1-D network.

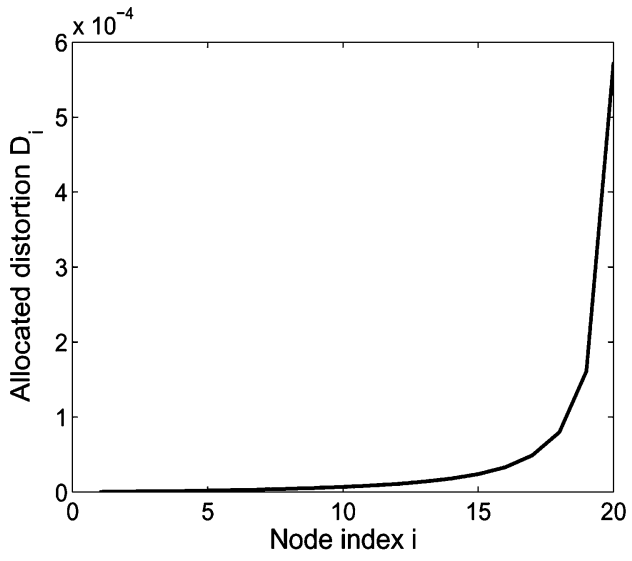

(a)

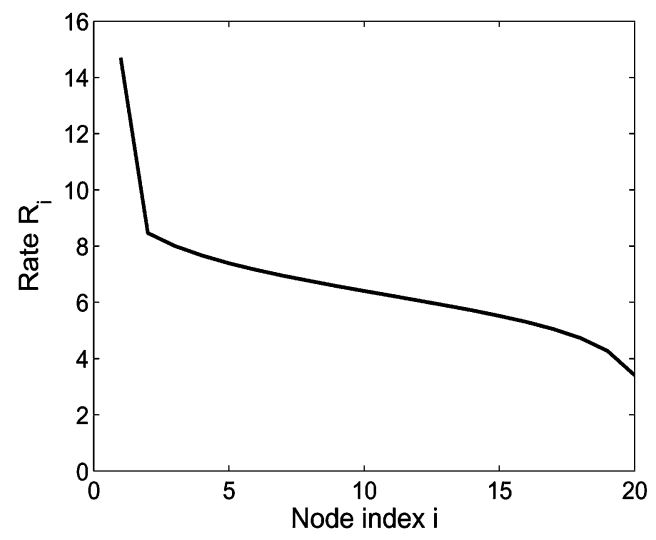

(b)

Fig. 8. Optimized placement for the 1-D network for the case of minimization of total consumed power. (a) Distortion allocation as a function of node index. (b) Rate allocation as a function of node index.

multidimensional convex in both $\left\{c_{i}\right\}_{i=1}^{N}$ and $\left\{R_{i}\right\}_{i=1}^{N}$, and the constraint sets are half hyperplanes for any fixed $\left\{D_{i}\right\}_{i=1}^{N}$.

The optimization in Step 2, a) of the Algorithm has been presented in Section III. We describe now the optimization in Step 2, b). For the sake of clarity, let us first ignore the internode constraints in (13). Denote $w_{i}^{\kappa}=c_{i}-c_{i-1}, i=1 \ldots N$, where $w_{i}$ is the Euclidean distance between node $i$ and node $i-1$, and $\kappa$ is the power coefficient of the distance ${ }^{4}$. Thus,

$$
c_{i}=\sum_{j=1}^{i} w_{i}^{2} .
$$

The values of $R_{i}^{*}$ are computed as in (10), with initial weights corresponding to equally spaced nodes $w_{i}=\frac{L}{N}$.

The expression of the solution for $R_{i}^{*}$ is independent of the actual value of the placement. Therefore, we can now rewrite the optimization (13) including the unknowns $\left\{w_{i}\right\}_{i=1}^{N}$ as follows:

$$
\left\{w_{i}^{*}\right\}_{i=1}^{N}=\arg \min _{\left\{w_{i}\right\}_{i=1}^{N}} \sum_{i=1}^{N}\left(\sum_{j=i}^{N} R_{j}^{*}\right) w_{i}^{\kappa}
$$

under the constraint:

$$
\sum_{i=1}^{N} w_{i}=L
$$

The solution of (14) is obtained using Lagrange multipliers. For $\kappa=2$, the closed-form solution is

$$
w_{i}^{*}=\frac{L}{\sum_{j=i}^{N} R_{j}^{*}\left(\sum_{l=1}^{N} \frac{1}{\sum_{j=l}^{N} R_{j}^{*}}\right)}, \quad i=1 \ldots N .
$$

${ }^{4}$ For the sake of the simplicity of analysis, we use $\kappa=2$ in solving the optimization problems, however, our results can be easily extended to arbitrary values of $\kappa$; it is hard to obtain closed-form solutions for arbitrary $\kappa>2$.
By placing the nodes as given by (15), the rate allocation changes as a function of $\left\{w_{i}^{*}\right\}_{i=1}^{N}$, since both the differential entropies and optimal distortions in (10) depend on the values of $c_{i}$. Next, for these values of $\left\{c_{i}^{*}\right\}_{i=1}^{N}$, we optimize over $\left\{R_{i}^{*}\right\}_{i=1}^{N}$ [Step 2, a) of Algorithm 1], and repeat this iterative procedure until the algorithm converges. The resulting distortion and rate allocation are shown in Fig. 8.

It is complicated to study in a closed-form the monotonicity of the rate allocation (10) for a placement which is not uniform, due to the complex interplay between node positions, distortion allocations and conditional differential entropies. However, our numerical simulations show monotonicity of the rate allocation in the case of power efficient optimal placement, as well.

For the instances we used ( $N=20, \alpha=10^{-3}, \kappa=2, \beta=1$, no constraints on the internode distances), the algorithm converges in at most four steps. Our numerical results show that nodes at the extremity of the network are largely spread, and nodes near the sink are closer to each other [see Fig. 9(b)].

Note that by using a similar technique we can solve the problem that additionally considers the constraints imposed on the maximum internode distances. In the resulting solution, the distances between the nodes would become more even.

\section{B. Network Lifetime Maximization}

The plot in Fig. 9 shows the power consumption at each node and the distances from the nodes to the sink for the scenario in Section IV-A, as a result of solving (13). Moreover, in Fig. 10, we plot the variance of power consumption per node as the size of network increases. We see that the decrease in variance is smaller as the size of the network increases.

The power consumption of a node is a quantity inversely proportional to the lifetime of a node. Sometimes, in practice, a different target of interest is to maximize the lifetime of the network. For instance, in Fig. 9, the last node $N$ will be the first to die. Let us now separately consider another set of constraints related to the lifetime of the network, 


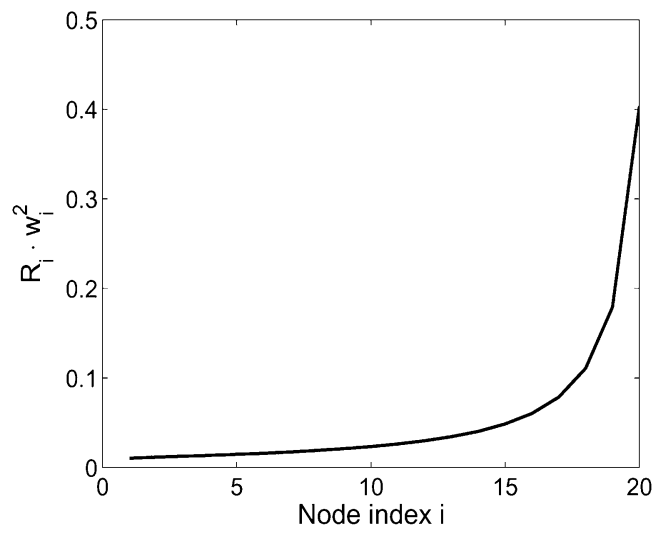

(a)

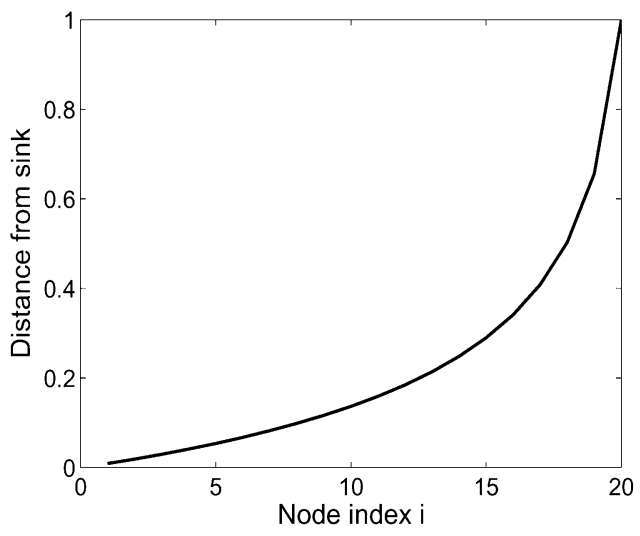

(b)

Fig. 9. Placement optimization for minimizing the total power. (a) Power consumption as a function of node index. (b) Distance from sink as a function of node index.

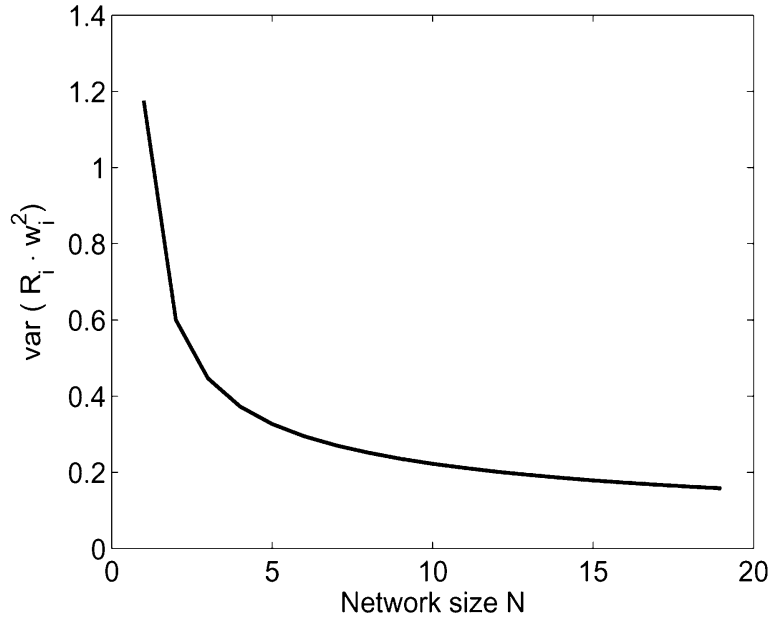

Fig. 10. Variance of the power consumption with the increase of the network size.

namely we impose that all nodes consume the same amount of power. This new set of constraints are aimed at maintaining a large number of nodes alive, which amounts to solving the following set of equations:

$$
\begin{aligned}
\sum_{i=1}^{N} w_{i} & =L \\
\left(\sum_{j=i}^{N} R_{j}^{*}\right) w_{i}^{2} & =\left(\sum_{j=k}^{N} R_{j}^{*}\right) w_{k}^{2}, \quad i, k=1 \ldots N \\
\sum_{i=1}^{N} D_{i} & =D
\end{aligned}
$$

and the solution is

$$
w_{i}^{*}=\frac{L}{\sqrt{\sum_{j=i}^{N} R_{j}^{*}}\left(\sum_{l=1}^{N} \sqrt{\frac{1}{\sum_{j=l}^{N} R_{j}^{*}}}\right)} .
$$

For an arbitrary value of the distance power coefficient $\kappa$, the square roots in (17) are replaced by the $\kappa$ th roots.

Note that $\left\{R_{i}^{*}\right\}_{i=1}^{N}$ depend on $\left\{w_{i}^{*}\right\}_{i=1}^{N}$ similarly to (10), thus, we can implement a similar iterative procedure to find the optimal weights $\left\{w_{i}^{*}\right\}_{i=1}^{N}$.

\section{Algorithm 2: Lifetime Optimization}

- until convergence do:

- (a) Given $\left\{w_{i}\right\}_{i=1}^{N}$, express $\left\{R_{i}\right\}_{i=1}^{N}$ as (10).

- (b) Solve (16) with parameters $\left\{R_{i}\right\}_{i=1}^{N}$ to obtain $\left\{w_{i}\right\}_{i=1}^{N}$ as (17).

Fig. 11 shows the distance from sink, and power consumption at each node, when the optimization of node placement is done as in (17).

For the same instance, the total power consumed with the optimized placement of Section IV-A is 1.17 [bit $\cdot \mathrm{m}^{2}$ ], whereas with the scenario here, the total power is 1.55 [bit $\cdot \mathrm{m}^{2}$ ]. Note that a placement that optimizes both the total power consumption of the network while maintaining the same individual consumption levels at nodes should be a tradeoff of the results of the two optimization problems.

However, it is complicated to provide an exact closed-form solution for the node placement that optimizes one of the target metrics under constraints on the other target metric. This requires starting from the node placement solution that optimizes one of the metrics, and computing continuously internode distances until the constraint on the other metric is satisfied. This optimization is clearly not scalable in network size, thus, we propose a simple suboptimal solution that still provides a good behavior, in terms of trading off both costs, by computing a weighted combination of the two separate placement solutions. Namely, if we denote the placement solution for total power minimization in (14) by $\left\{w_{i}^{\prime}\right\}_{i=1}^{N}$, and the placement solution for network lifetime maximization in (17) by $\left\{w_{i}^{\prime \prime}\right\}_{i=1}^{N}$, then our proposed tradeoff solution is

$$
w_{i}^{*}=\gamma \cdot w_{i}^{\prime \prime}+(1-\gamma) \cdot w_{i}^{\prime}, \quad \gamma \in[0,1], \quad i=1 \ldots N
$$

In Fig. 12, we plot the normalized values for the total power and respectively power variance when node positioning is done as in (18). Note that, as expected, the choice of $\gamma$ provides a tradeoff between having a small power consumption for the network versus equalizing the individual power at nodes.

\section{CONCLUSION}

We considered the problem of power efficient data gathering in sensor networks, with high-resolution coding, under distortion constraints. We found the rate and distortion allocations in a closed-form, and illustrated our results with numerical experiments on Gaussian correlated random fields. We also studied the problem of optimal placement for two power efficiency targets of interests, namely total 


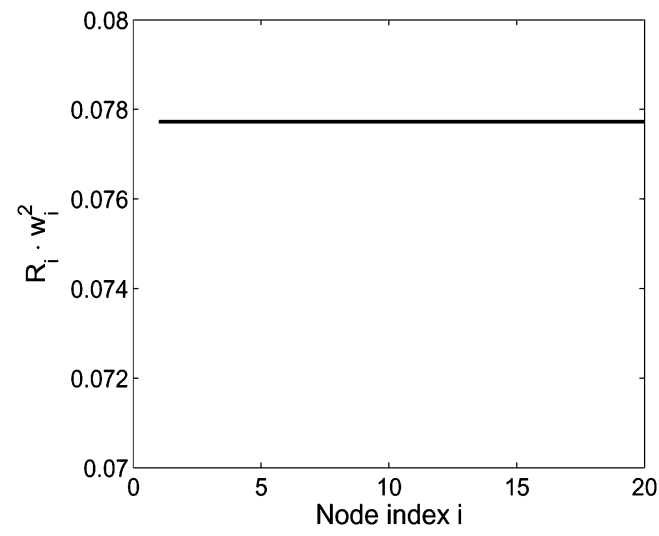

(a)

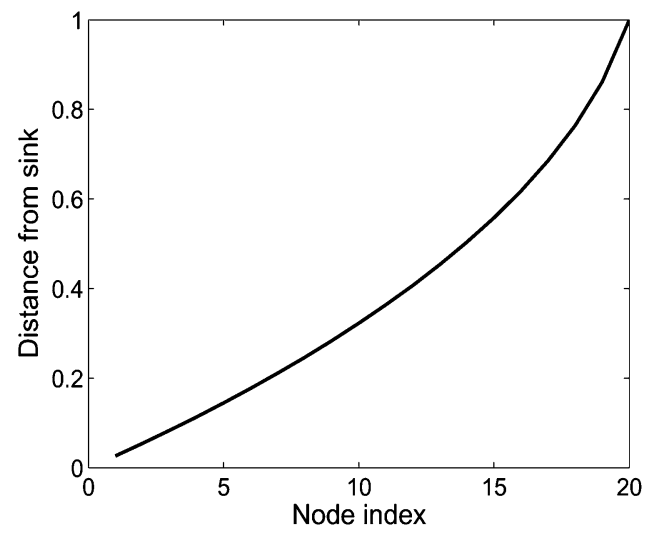

(b)

Fig. 11. Placement for lifetime optimization. (a) Power consumption as a function of node index. (b) Distance from sink as a function of node index.

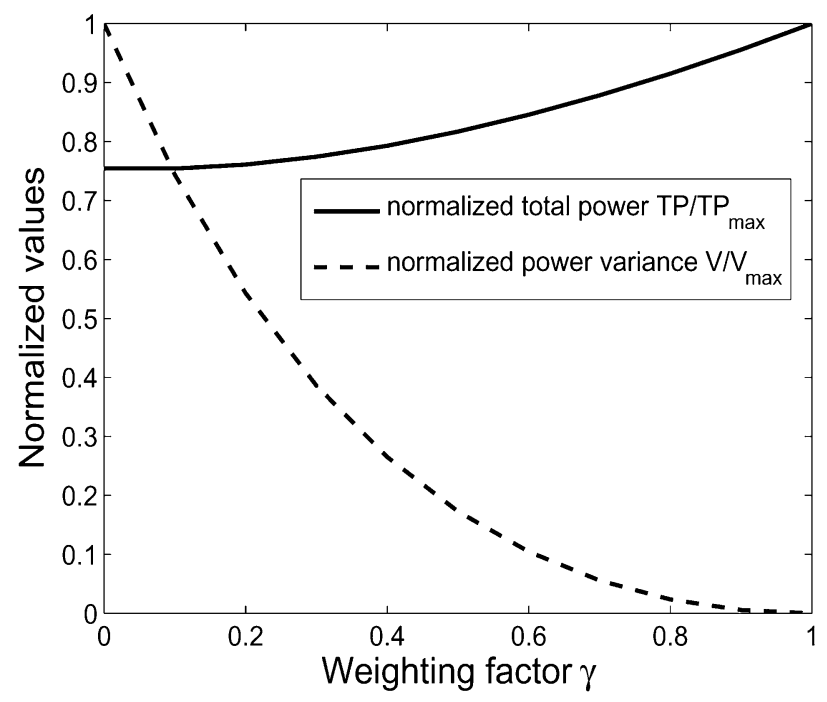

Fig. 12. Normalized total power and power variance for the tradeoff placement, as a function of the weighting factor $\gamma$.

power and network lifetime, and compared the tradeoffs involved. Finally, we proposed a node placement solution that provides a tradeoff for the optimization of the two metrics.

\section{ACKNOWLEDGMENT}

The authors wish to thank M. Vetterli for useful discussions.

\section{REFERENCES}

[1] S. Arora, "Polynomial time approximation scheme for euclidean TSP and other geometric problems," in Proc. 37th Ann. IEEE Symp. Found. Comput. Sci., 1996, pp. 2-11.

[2] J. Barros, C. Peraki, and S. D. Servetto, "Efficient network architectures for sensor reachback," in Proc. IEEE Int. Zurich Seminar on Commun., 2004.

[3] T. Berger, Multiterminal Source Coding: Lectures presented at CISM Summer School on the Information Theory Approach to Communications, 1977.

[4] D. Bertsekas, Network Optimization: Continuous and Discrete Models. Belmont, MA: Athena Scientific, 1998.

[5] C. Chong and S. P. Kumar, "Sensor networks: Evolution, opportunities and challenges," Proc. IEEE, vol. 91, no. 8, 2003.

[6] T. M. Cover and J. A. Thomas, Elements of Information Theory. New York: Wiley, 1991.
[7] N. Cressie, Statistics for Spatial Data. New York: Wiley, 1991.

[8] R. Cristescu, B. Beferull-Lozano, and M. Vetterli, "Networked SlepianWolf: Theory, algorithms and scaling laws," IEEE Trans. Inf. Theory, vol. 51, no. 12, pp. 4057-4073, Dec. 2005.

[9] R. Cristescu, B. Beferull-Lozano, M. Vetterli, and R. Wattenhofer, "Network correlated data gathering with explicit communication: NP-completeness and algorithms," IEEE Trans. Netw., vol. 14, no. 1, pp. 41-54, Feb. 2006.

[10] R. Cristescu, B. Beferull-Lozano, and M. Vetterli, "On network correlated data gathering," in Proc. INFOCOM, Hong-Kong, China, 2004.

[11] M. Enachescu, A. Goel, R. Govindan, and R. Motwani, "Scale free aggregation in sensor networks," Algosensors, 2004.

[12] M. Franceschetti, J. Bruck, and L. J. Schulman, "A random walk model of wave propagation," IEEE Trans. Antennas Propag., vol. 52, May 2004.

[13] D. Ganesan, R. Cristescu, and B. Beferull-Lozano, "Power-efficient sensor placement and transmission structure for data gathering under distortion constraints," ACM Trans. Sensor Netw., 2005, to be published.

[14] A. Goel and D. Estrin, "Simultaneous optimization for concave costs: Single sink aggregation or single source buy-at-bulk," in Proc. ACMSIAM Symp. Discrete Algorithms, 2003, pp. 499-507.

[15] D. Marco, E. Duarte-Melo, M. Liu, and D. L. Neuhoff, "On the many-to-one transport capacity of a dense wireless sensor network and the compressibility of its data," in Proc. IEEE/ACM IPSN, Palo Alto Research Center (PARC), Palo Alto, CA, 2003.

[16] A. Pandya, A. Kansal, G. Pottie, and M. Srivastava, "Bounds on the rate-distortion of multiple cooperative Gaussian sources," Univ. Calif. Los Angeles, Tech. Rep., 2003.

[17] S. Pattem, B. Krishnamachari, and R. Govindan, "The impact of spatial correlation on routing with compression in wireless sensor networks," in Proc. IPSN, Berkeley, CA, 2004.

[18] G. J. Pottie and W. J. Kaiser, "Wireless integrated sensor networks," Commun. ACM, vol. 43, no. 5, pp. 51-58, 2000.

[19] D. Slepian and J. K. Wolf, "Noiseless coding of correlated information sources," IEEE Trans. Inf. Theory, vol. IT-19, pp. 471-480, 1973.

[20] R. Zamir, "The rate loss in the Wyner-Ziv problem," IEEE Trans. Inf. Theory, vol. 42, pp. 2073-2084, Nov. 1996.

[21] R. Zamir and T. Berger, "Multiterminal source coding with high resolution," IEEE Trans. Inf. Theory, vol. 45, pp. 106-117, Jan. 1999. 